\title{
Tuning of the ultra slow muon beamline by utilizing ionized hydrogen
}

\author{
Taihei Adachi' ${ }^{1}$, Yutaka Ikedo, Atsushi Yabuuchi, Patrick Strasser, \\ Kenji M. Kojima, Shunsuke Makimura, Jumpei Nakamura, Koichiro Shimomura, \\ Yasuo Miyake \\ High Energy Accelerator Research Organization (KEK) \\ 1-1 Oho, Tsukuba, Ibaraki 305-0801, Japan \\ E-mail: adachitepost.kek.jp
}

Takashi Nagatomo

Nishina Centre, RIKEN

Hirosawa 2-1, Wako, Saitama 351-0198, Japan

\section{Wataru Higemoto, Takashi U. Ito}

Advanced Science Research Centre, Japan Atomic Energy Agency

Shirakata-shirane 2-4, Tokai, Ibaraki 319-1195, Japan

E-mail: higemoto.wataru@jaea.go.jp

\section{Eiko Torikai}

University of Yamanashi

Takeda 4-3-11, Kofu, Yamanashi 400-8511, Japan

E-mail: et@yamanashi.ac.jp

\begin{abstract}
The ultra-slow muon beam is expected to be a new probe for surface and interface physics. A new beamline for the ultra-slow muon is now under construction at the J-PARC. Before transporting ultra-slow muons through the transport system, we tuned the system utilizing laser ionized hydrogen ions as ultra-slow beam. We successfully transported ions to the end of the beam line. Optimization of the beam transport system is in progress to be ready for generating the ultra-slow muon beam.
\end{abstract}

16th International Workshop on Neutrino Factories and Future Neutrino Beam Facilities 25 -30 August, 2014

University of Glasgow, United Kingdom

\footnotetext{
${ }^{1}$ Speaker

(C) Copyright owned by the author(s) under the terms of the Creative Commons Attribution-NonCommercial-ShareAlike Licence.
} 
Introduction

Muons are unique probe for both condensed matter physics and particle physics, especially useful for investigating local magnetic fields in materials by using the muon spin rotation, relaxation and resonance technique $(\mu \mathrm{SR})$. The ultra-slow muon beam with small beam radius and narrow energy spread is expected to extend the $\mu \mathrm{SR}$ from bulk materials to tiny or thin samples, surfaces, and interfaces research. The first experiment for generating ultra-slow muon beam was conducted at the Meson Science Laboratory of KEK in 1995 [1, 2]. Improved experiment has been carried out at RIKEN-RAL [3]. A new beam line, which aiming the thousands times higher intensity of ultra-slow muon beam than the intensities of previous beam lines, is now under construction and commissioning at J-PARC.

\section{The Ultra-Slow Muon Transport System}

The new beam line, called the U-Line, has been installed in the Materials and Life Science Experimental Facility (MLF) of the J-PARC. A schematic view of the U-Line is shown in Fig. 1 (left). The muons are produced by the $3 \mathrm{GeV}$ proton beam hitting a muon production target consist of graphite. The repetition rate of the proton beam is $25 \mathrm{~Hz}$. Each proton pulse consist of two bunches separated by 600 ns. One bunch has a full width of 100-120 ns. The produced muons, especially surface muons $(\sim 4 \mathrm{MeV})$ are captured and transported mainly by magnetic field of solenoids. The large solid angle of acceptance can be attained with use of MIC Capture Solenoid. Wide special and momentum acceptance, which comes from large bore and high magnetic fields of solenoids, allows us to extract high intensity muon beam to the experimental hall [4]. The layout of the new ultra-slow muon transport system is shown in Fig. 1 (Right). The

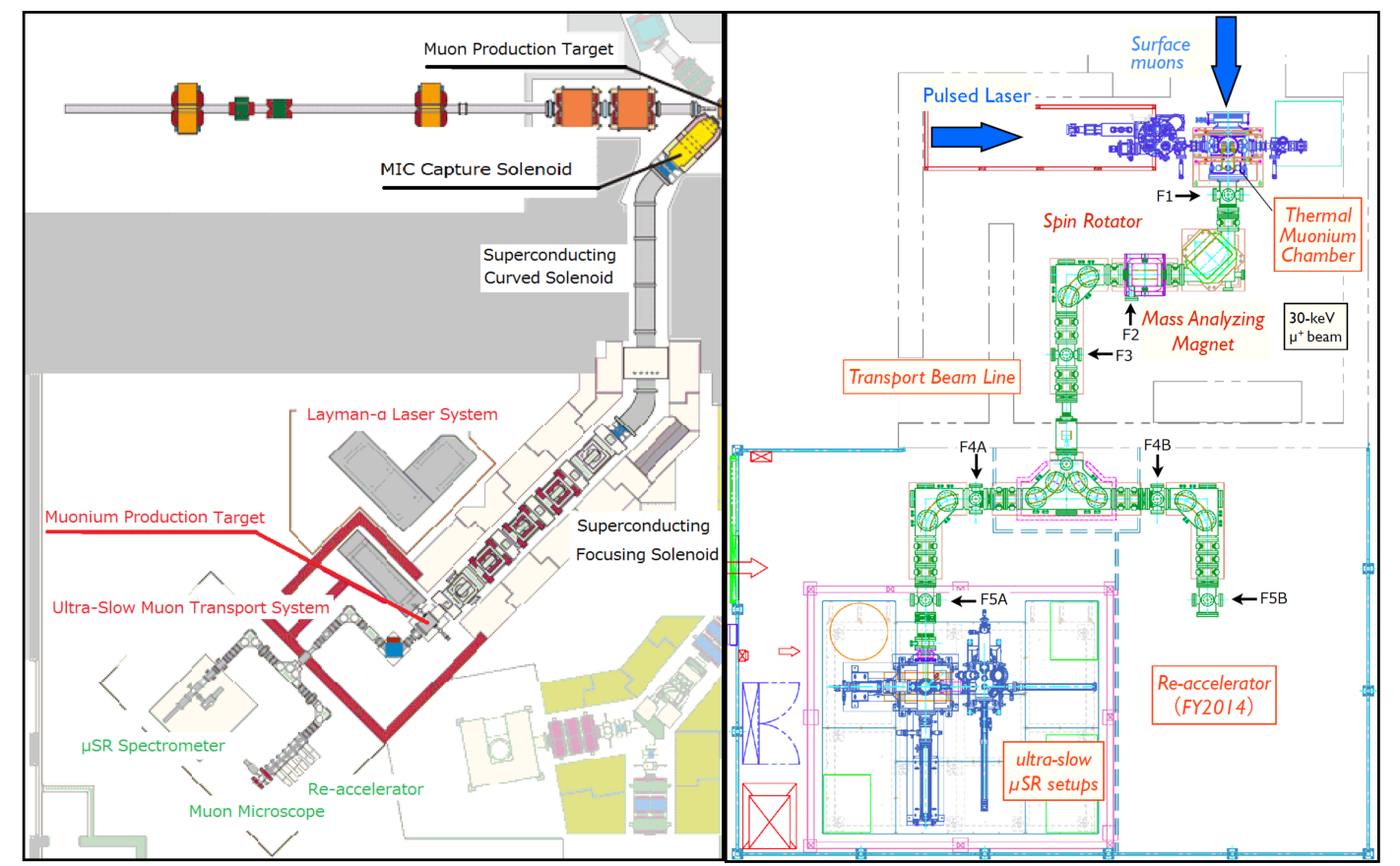

Fig. 1. (Left) A schematic view of the U-Line at the MLF. (Right) The layout of the Ultra-Slow Muon Transport System. F1 F5 show focusing points of the beam line. 
surface muon beam is stopped in a hot tungsten target and evaporate in to vacuum as muoniums. The muoniums are ionized by pulsed laser and become the ultra-slow muon. A SOA lens, which is located downstream of the tungsten target, extract and accelerate muons up to $30 \mathrm{keV}$. A series of electric quadrupoles, magnetic bend, and electric bends transport muons to experimental area [5]. Almost all of the components of the system has been installed [6]. One branch of the ultraslow muon beam line for re-accelerator experiments has not finished.

\section{The tuning by utilizing laser ionized hydrogen}

Before transporting ultra-slow muons through the transport system, we tuned the system utilizing laser ionized hydrogens as ultra-slow beam. Hydrogen is the lightest atom and has only one electron. So, it is close to muonium. Hydrogens are contained in residual gases as molecules. On the surface of the hot tungsten target, single hydrogen atoms are produced from the hydrogen molecules. Then hydrogen atoms diffuse in to vacuum. Ionization lasers are injected into the downstream of the tungsten target, and ionize the hydrogen atoms as is the case of the muons.

Laser ionized hydrogens are successfully transported to the $\mu$ SR chamber, which is an end of beamline. Micro Channel Plates (ROENDEC DLD40) are inserted into beamline to measure the rate of particles. The measured time of flight (TOF) between the laser injections and the particle detections are shown in Fig. 2. The measured TOF agrees with calculated TOF of hydrogen ion. The tuning by utilizing laser ionized hydrogen is currently underway.

\section{Summary}

Installation of the ultra-slow muon beam line was almost completed at J-PARC. Laser ionized hydrogen-ions as pilot beam were successfully transported to the ultra-slow $\mu \mathrm{SR}$ chamber. The tuning is currently underway for the first ultra-slow muon beam at J-PARC.
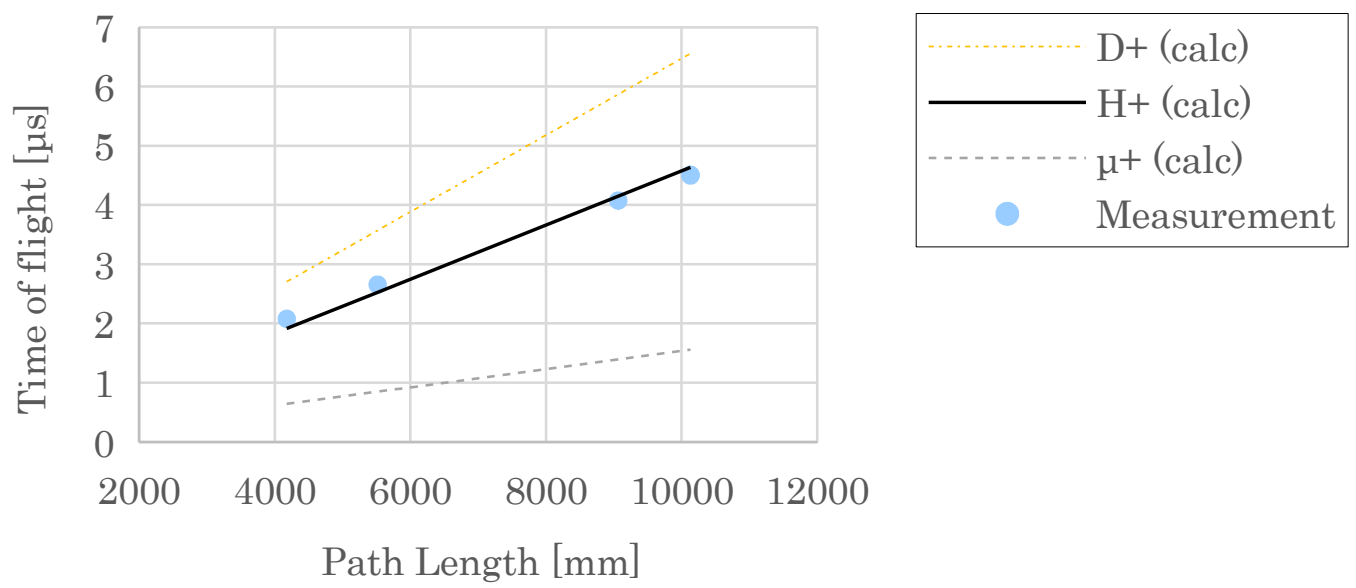

Fig. 2. Time of flight of the beam. Dots shows the results of measurements. Lines show calculated TOF of muon, hydrogen-ion, and deuterium-ion. 


\section{Acknowledgment}

This work was supported by a Grant-in-Aid for Scientific Research on Innovative Area "Frontier of Materials, Life and Particle Science Explored by Ultra Slow Muon Microscope" (No.23108002) of the Ministry of Education, Culture, Sports, Science, and Technology, Japan.

\section{References}

[1] K. Nagamine et al., Phys. Rev. Lett. 74 (1995) 4811.

[2] Y. Miyake et al., Nucl. Instr. B95 (1995) 265

[3] P. Bakule et al., Nucl. Instr. B266 (2008) 335

[4] Y. Ikedo et al., JPS Conf. Proc. 2 (2014) 010103

[5] P. Strasser et al., MLS-Report 2011 (2012) 14

[6] T. Nagatomo et al., JPS Conf. Proc. 2 (2014) 010102 